\title{
Road rehabilitation as an opportunity to analyse the approach in designing pavement constructions
}

\author{
Slavoljub Erjavec ${ }^{1, *}$, Slobodan Ognjenovic ${ }^{2}$, Zlatko Zafirovski ${ }^{2}$ \\ ${ }^{1}$ Capp-Lay, Belgrade, Serbia \\ ${ }^{2}$ Saints Cyril and Methodius University of Skopje, Skopje, Macedonia
}

\begin{abstract}
The pavement constructions of highways built in he eighties mainly featured low bearing surfaces with cement stabilization and asphalt layers with the total thickness of up to $26 \mathrm{~cm}$. The nineties were a period of rationalization, mainly referring to the reduction of the asphalt layer thickness. This paper will compare the two approaches upon the designing of pavement constructions in Serbia and in Macedonia. The paper presents subjects dealing with issues like the choice between the "long-term" or "rationalized" pavement construction as well as with problems in the evaluation of layers of non-cohesive granulated material or the layer of cement-connected stone material. Contrary to this, the present paper does not deal with questions related to numerous diagnostic methods applied in the assessment of the pavement conditions, which is otherwise a rather important segment in the process of road rehabilitation design.
\end{abstract}

\section{The concept of a long-term pavement construction}

The pavement constructions of the highways built in the eighties were designed with: (1) a $20 \mathrm{~cm}$ cement-stabilized bearing layer nand 2) asphalt layers with the total thickness of 26 $\mathrm{cm}$.

The designing and the building of the pavement constructions in the nineties experienced a period of ratitonalization of design solutions, primarily reflected in the reduction of the thickness of asphalt layers. The practice of reduction of the thickness of the asphalt layers in planning and design continued during the first two decades of the $21^{\text {st }}$ century so that this changed approach was applied in the last stage of the highway construction on the corridor $\mathrm{X}$, which means that the mixed-type pavement constructions included the building of asphalt layers of the $17 \mathrm{~cm}$ overall thickness of while in highways where the building of a flexible type pavement construction was envisaged, and where the thickness of the asphalt layers was $18 \mathrm{~cm}$ to $22 \mathrm{~cm}$. It can be noticed that the approach applied during the eighties is now recognized as "high initial investment strategy" or "longterm pavement" (The European Long-Life Pavement Group (ELLPAG) was subsequently established as a FEHRL Working Group to act as the focal point for determining the way forward. Members of the Group comprise representatives of research institutes (FEHRL

*Corresponding author: slavoljub.erjavec@gmail.com 
members) and the UK Highways Agency, representing CEDR.). The benefits of this approach reflect in amortization of the following risks: (1) the risk of unplanned increase in the scope and aggressivity of cargo traffic (This opens the question of the design life of the pavement construction as well as the one of a referent axis which, according tot $\mathrm{h}$ eauthor, considerably influence the choice of design solution), (2) the risk including the discrepancies of the ideal model of calculation - dimensioning with the pavement decay mechanism during road exploitation which includes insufficient connection between the demanded characteristics of the materials and the parameters applied in designing, (3) the risk of errors incurred during the design, (4) the risk of |relaxation| of the material specifications in the process of the choice of material, and finally the maintenance of the road and pavement. This approach leads to the fact that, in the long exploitation period, and in the cycle of periodical maintenance-rehabilitaiton of the pavements, the interventions are reduced to the improvement of pavement functionality while the structural characteristics of the dominant length remain intact, with a high percentage of residual value.

The sections of the E-70 and E-75 highways, on which the condition of the last rehabilitation cycle was evaluated is illustrated by the two presented approaches. Besides the plastic deformations which predominantly end in the wear layer cracks also appear as a consequence of aging of the material, influences which are not related to traffic load. Especially noticeable are the transversal cracks reflected from the cement-stabilized layer, which can be considerd in the context of "innate defects" in the context of "life with cracks" approach. Finally, as expected, the analyses of the fatigue cracks, the deflection analysis and the analyses of the embedded materials confirmed that the residual value is on a high level with only occasional, local recognition of the bearing capacity loss, mainly rigidity of asphalt layers.

On sections where the pavement construction has been designed as per the strategy of low initial investments, material fatique cracks, accompanied by deformations, appear as early as after the first exploitation period. The bearing layers have a low remaining value, therefore the damage and the low comfort is related to the low bearing capacity. The available design measures often include interventions in the bearing layers or offer a coldprocedure deep recycling as an alternative.

\section{Assessment of the non-cohesive granulated material level}

The level to which the granulometric composition and the plasticity of the "minuscule" particles are responsible for the damage of the asphalt layers is a question asked upon every assessment of the pavement construction condition. Numerous laboratory and field analysis are available. In laboratory conditions it is possible to establish all three basic components indicating the quality of the used material: (1) the granulometric composition parameters, the maximal grain dimensions, the grain form and the $\mathrm{Cu}$ and $\mathrm{Cc}$ parameters, (2) the content and plasticity of the "minuscule" particles - wl, IP, sand equivalent and possibly the methylene blue and (3) solidity, tendency to crushing and the permanence in conditions of increased humidity. The field research usually includes the analysis of plate bearing capacity, the field CBR, DCP and the compactness of the layer.

Upon the condition assessment we often come across the situation of leaching of the noncoherent granulated material immediately underneath the asphalt and of a filler connected, to a larger or smaller level, by the grains of the stone material. As we have already mentioned, the laboratory conditions allow us to determine the content and the quality of the minuscule particles, which we do. Although we decided and accepted certain criteria of evaluation of acceptability of the contents and plasticity, we are actually not familiar with the fundamental parameters of that material. 


\subsection{Theoretical basis}

The point is that not the permanent, but the elastic deformation of certain layers appears in the models of calculation of stress and dilatation meaning that the elastic module is the basic design parameter. On the other hand, the nature of the non-cohesive stone material manifests a tendency towards a permanent, plastic deformation.

That unwanted plastic deformation should be avoided or reduced to a minimum. In the case of the non-coherent granulated material layer, there is a critical stress level at which the elastic behavior is transformed into plastic deformation exists for every material type. If permanent deformation subsides during a long period of road exploitation it can be said that the layer of con-coherent granulated material bears a slight risk of permanent deformation.

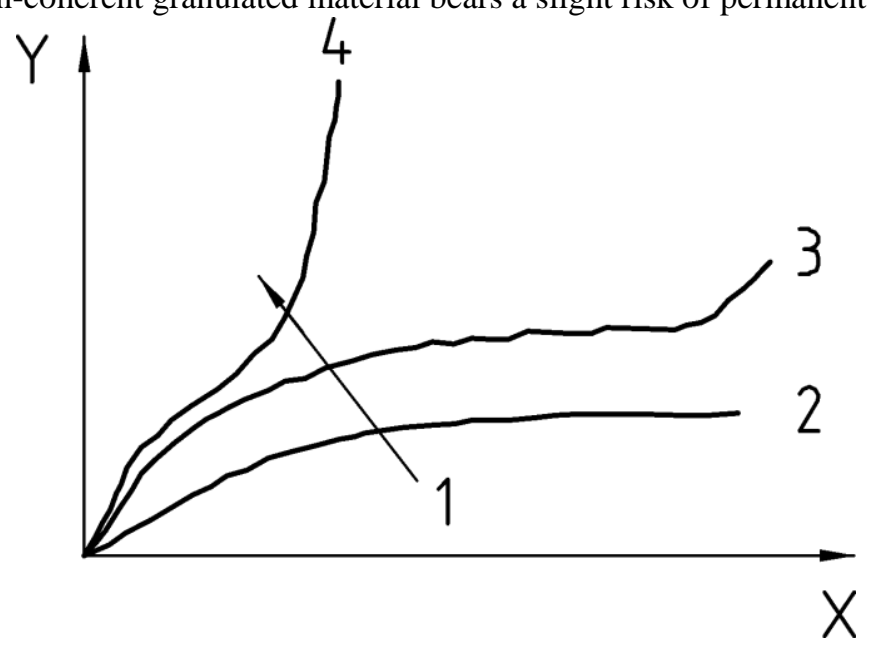

Fig. 1. Plastic behavior of non-coherent granulated material. Legend: 1 - increase of solidity deviator, 2 - stable, elastic behavior after a long cycle of repeated load, 3 - plastic deformation after a long cycle of repeated load, 4 - plastic deformation after a short cycle of repeated load. $\mathrm{X}$ - number of cycles of repeated load, $\mathrm{Y}$ - plastic deformation.

From the designer's point of view, if the deformation tends towards elastic behaviour than the calculation more closely reflects the behavior of the material in the construction. What is also important for the designing or modelling is whether the plastic deformation results upon small or high number of repeated loads, if any such risk is identified. A closer comprehension of the stress condition upon which the layer of non-coherent granulated material starts to receive a considerable increase of plastic deformation, whether this happens upon a small or a high number of repeated load, can help in the formation of a more reliable model of decay of the non-coherent granulated material and thereby provide a more reliable assessment of the individual options in the design of pavement rehabilitation. Namely, it can help in deciding whether this and above layers have to be removed or there is a small damage risk. 


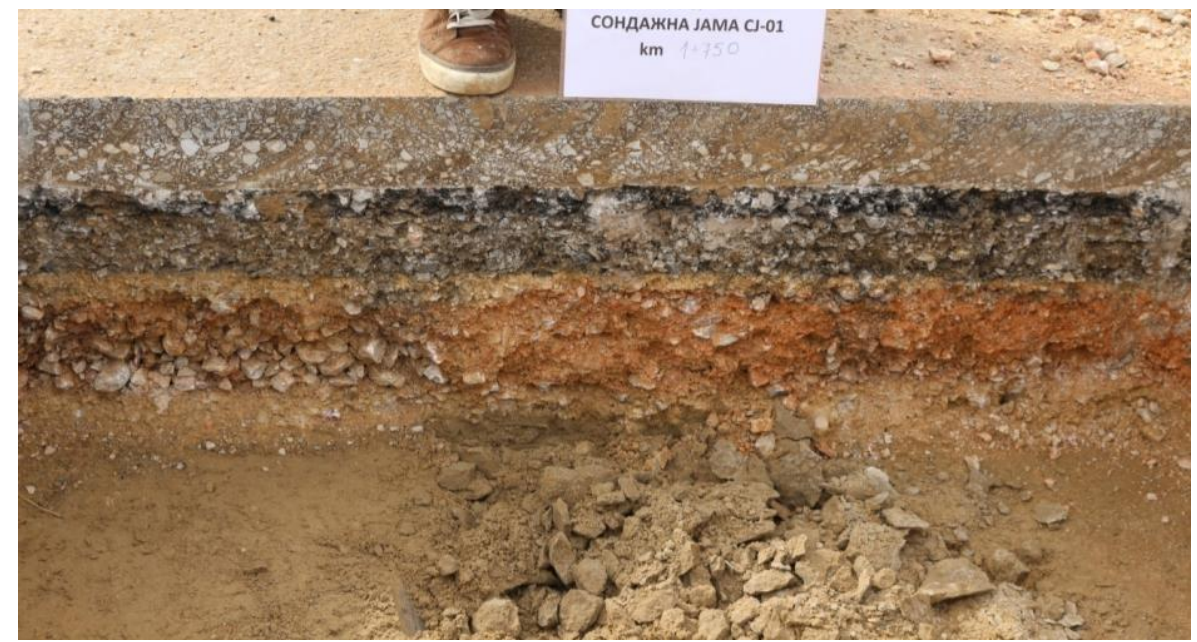

Fig. 2. Pavement construction profile in a test pit.

\subsection{Evaluation of the minuscule particle content}

Thje actual knowledge indicates that the quality and the quantity of „minuscule“ particles have one of primary influences on the features of the non-coherent granulated material layer. It is a common opinion that if the layer of the non-coherent granulated material contains less than $3 \%$ of ,minuscule particles“, it is not necessary to analyze the influence of the minuscule particles by one of the laboratory methods. On the other hand, if the layer of non-coherent granulated material contains more than $12 \%$ of „minuscule“ particles, there is no question about its negative impact. Control upon this important issuer is established if the use of minuscule particles is limited to the use of an inert, clay-free filter.

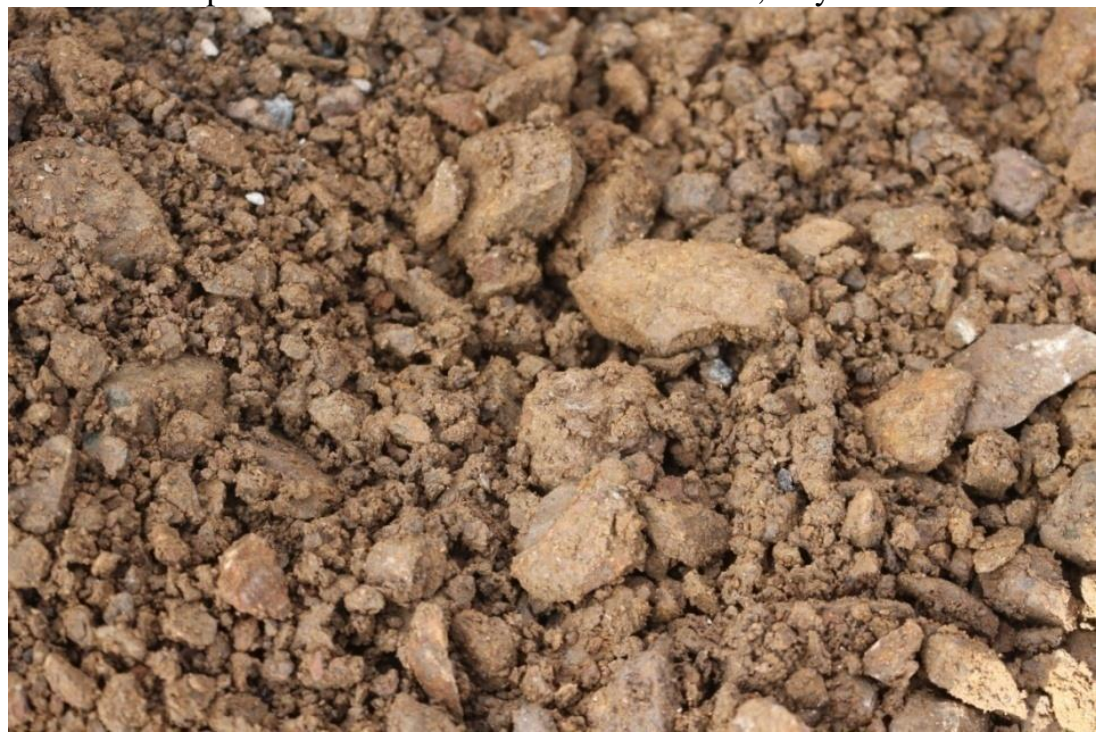

Fig. 3. Non-coherent granulated material sampled from the test pit $-D_{\max }=31 \mathrm{~mm}$.

However, there are the following situations that are very frequently encountered and need an answer: (a) is it possible, on a certain road, to apply non-coherent granulated material containing clayish particles and which quantity and plasticity leave a small risk of 
damage upon the designed stress condition, (b) is it justifiable to redo the pavement construction if any clayish particles are identified?

\subsection{Layer modelling - design problems}

If it is concluded that the layer of non-coherent granulated material is not of appropriate quality, that is is necessary, as in compliance of the rehabilitation methodology (DMRB Volume 7, Section 3, Part 3, Maintenance Assessment Procedure-HD 30/08, Strana), to reconsider the justifiability of the removal of the respective layer. Deciding between the removal of the layer of non-coherent granulated material and the construction of a reinforcement, implying retention of this layer as well, requires the answer to the question of whether the respective material tends to "subside" plastic deformation or not, that is, whether it tends to a progressive collapse for the existing - designed stress condition.

This raises the question of the consequence of the retention of the existing material in the pavement construction. In order to perform this evaluation, it is necessary, in the process of formation of the model of pavement decay, for the calculation of the permanence and prediction of the influence of the non-coherent granulated material layer on the decay of the pavement construction and possess data on the fundamental features of the respective layer material (elastic and plastic deformation, layer module). In other words, rutine laboratory analyses and the field analyses do not offer a detailed response about whether it is possible to expect any progressive increase of plastic deformation. Therefore it is necessary to establish a corelation between the said field analyses with the fundamental characteristics of the materials.

\section{Cement stabilized bearing layer}

In the course of the 80ties the highway pavement constructions have the bearing layer of cement-stabilized sandy gravel. A reliable method of determination of the rigidity of this layer was established in the latest cycle of road rehabilitation and during the current program. It is now already possible to say that, when possessing reliable data about te thickness of primarily the asphalt layers which can, on highways, be provided even without a georadar, the return calculation of the module on the basis of the measured deflections, and the application of the linear elastic theory gives a clear picture of the rigidity and the space homogeneity of the cement-stabilized sandy gravel layer.

\subsection{Evaluaiton of the bearing capacity through evaluation of rigidity}

As already mentioned, the high coordination of the rigidity model of this layer, achieved by the return calculation on the basis of deflections measured by the FWD appliance Dynatest and the compactness of the layer evaluated in the pits usually opened on around $1.0 \mathrm{~km}$ distance was proved in two rehabilitation cycles.

The following principle is applied upon the condition evaluation: 


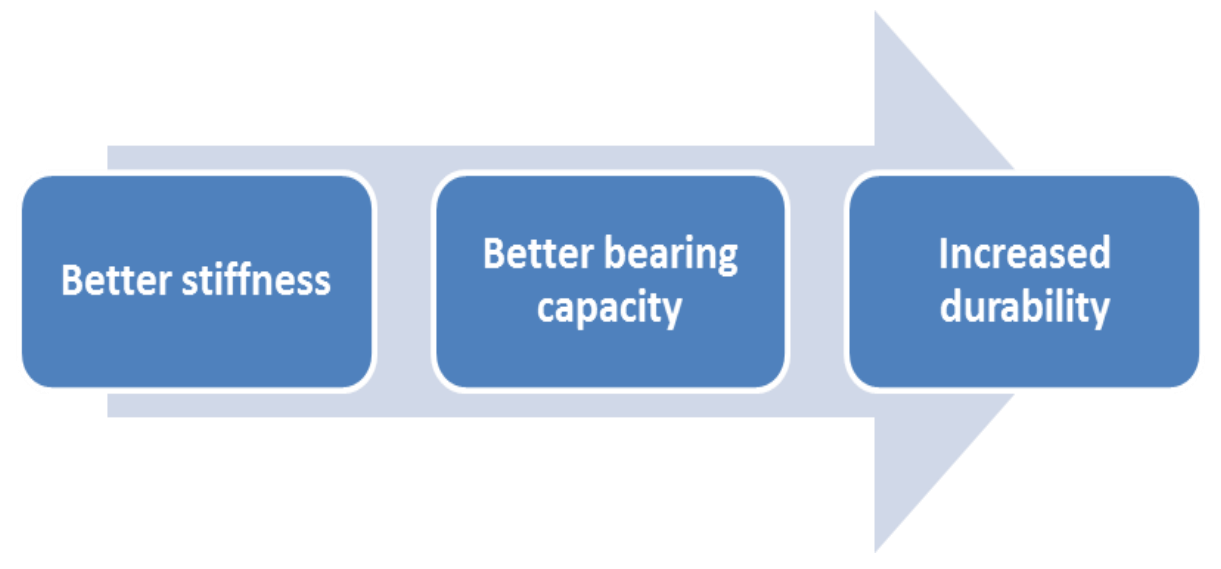

Fig. 4. The principle applied upon the condition evaluation.

The said principle is supported by the instruction on the evaluation of the condition of the British Highway Agency (DMRB - Volume 7, Section 3, Part 3, Maintenance Assessment Procedure-HD 30/08), the French instruction on designing of pavement constructions (CONCEPTION ET DIMENSIONNEMENT DES STRUCTURES DE CHAUSSÉE - Guide technique LCPC, SETRA, Paris 1994. (page 112)) and the European standard of EN 14227-1 dealing with the quality assessment of the cement stabilized material.

On the basis of the experiences acquired in the previous and in the latest cycle of pavement rehabilitation, we present a summary description of the structure of a mixed type pavement construction. The structure is described in 6 condition types. The principal decisive factor is the rigidity of the asphalt and the cement-stabilized layer, as well as the damage of the pavement surface. Additionally to the description, these types can have damages treated in the same ways as when the construction of the flexible type construction is in question, including rutting, bitumen leaching, surface cracks, removal of aggregates (See Table 1). 
Table 1. Condition classes of the rigid layers of a mixed-type pavement construction.

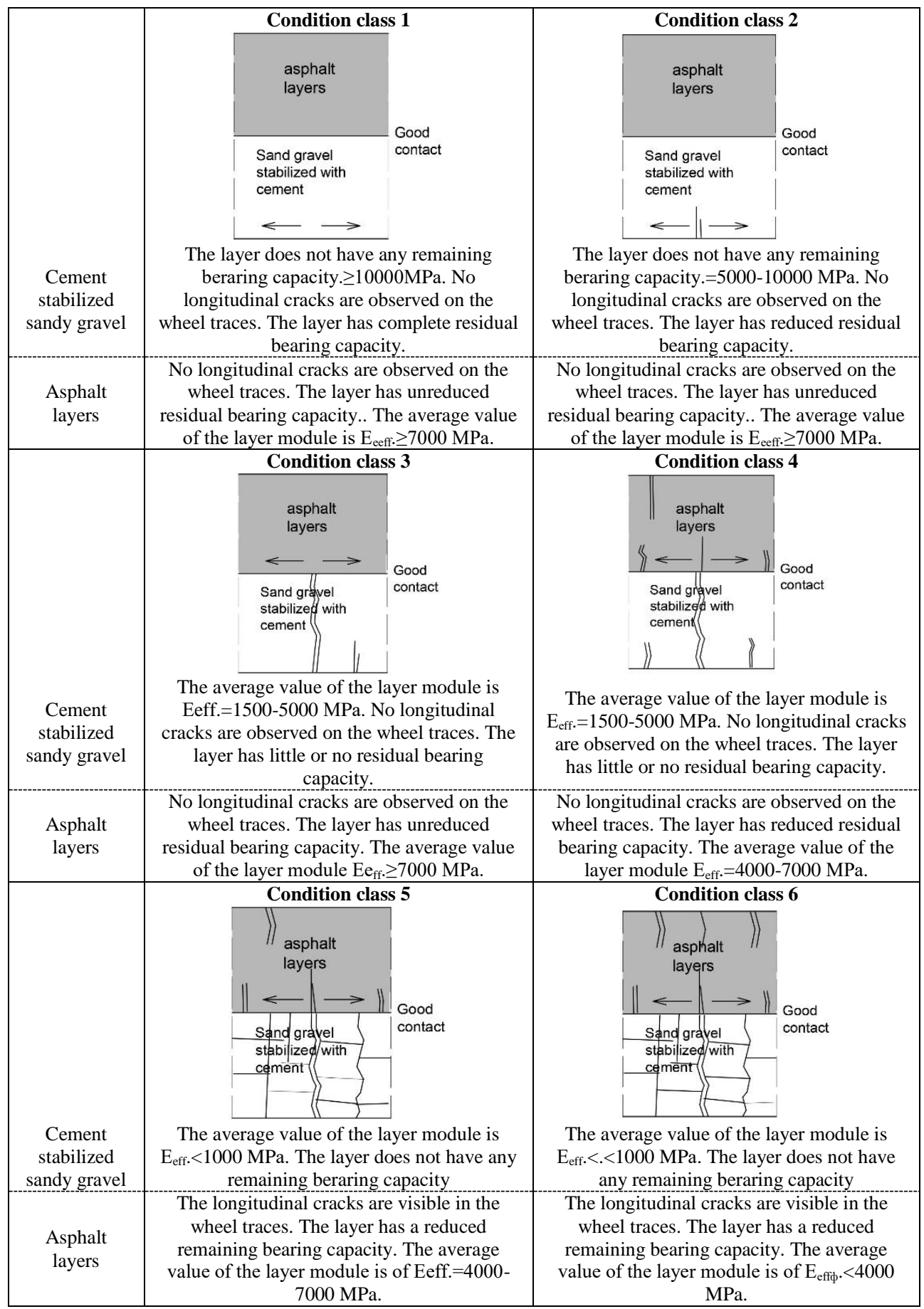




\subsection{Omission of the phase of high bearing capacity with the stabilized layer cement}

It has to be mentioned that there is an approach different from the described one. It relies on the thesis that the crack reflection should be avoided bu designing and performing a layer of a low-rigidity layer, which can be accomplished by application of the appropriate hydraulic binder or by formation of cracks in the early stage of layer formation. This approach better emphasizes the reduction of risk of the formation of transversal cracks in this layer and its further reflection in relation to the high bearing capacity which remains ineffective in this way. This in fact means that this layer and pavement construction as a whole are missing for the first stage of the bearing capacity (see figure number 6).

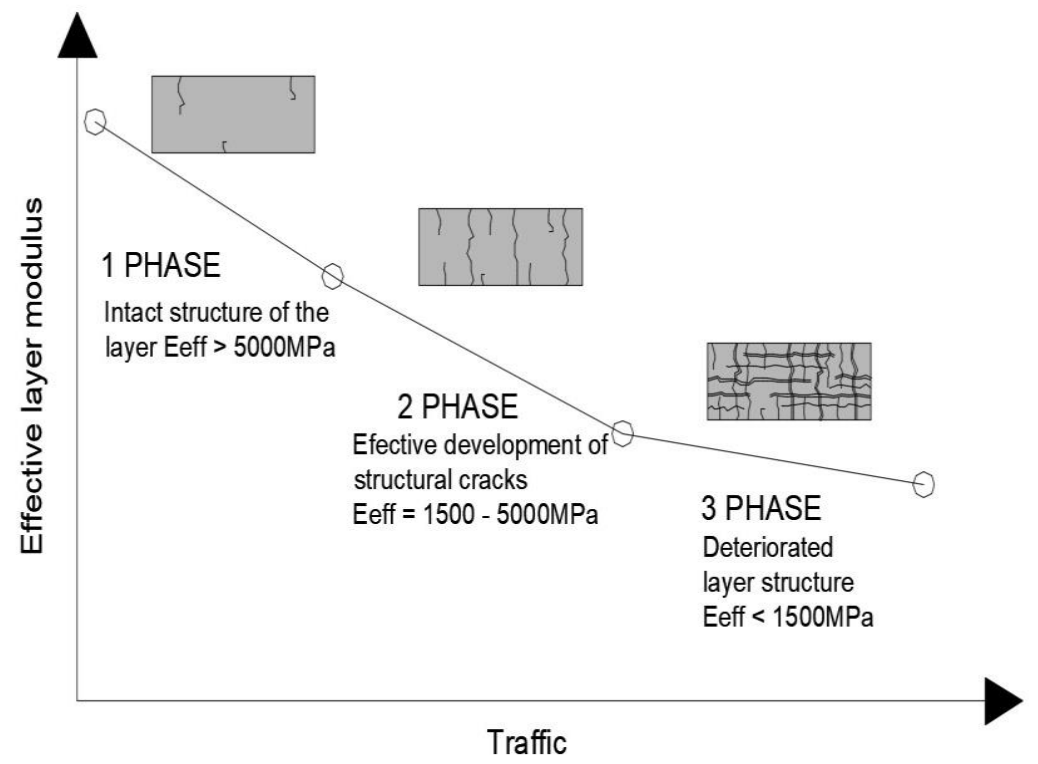

Fig. 5. Idealized model- basic phases in the process of damaging of the cement-stabilized granulated material layer.

The designer is expected to carefully select the calculation model. The models used for the calculation of permanence of the pavement construction with cement-stabilized granulated material of high rigidity are not applicable in the calculation of the permanence of the pavement constructions with a bearing capacity based on the low-rigidity concept.

\subsection{Reflected cracks}

Reflected transversal cracks are not avoided, on the contrary. They are the "logo" of a pavement construction with a bearing layer of cement-stabilised granulated material. The measures usually applied prolong the reappearance but life with reflected cracks goes on. However, it is encouraging that the measuring of the load transfer on cracks, on highway sections subject to evaluation, has led to the conclusion that average value of the LTE parameter is stable over $85 \%$ and the pavement delevelling exceeds the value of $5 \mathrm{~mm}$ only in individual cases. What is somewhat more worrying and requires more intensive attention of engineers is the development of transversal cracks in whose environment develops the crack network. Such cracks are formed on the contact of two surfaces, the surface of the layer with a loosely bound material and the one where high solidity was accomplished. Such cracks are accompanied by bilateral slope in the zone of the crack itself. 
Eminent road laboratories have decade-long experience in evaluation and selection of measures to attenuate the crack reflection. Experiences regarding the reduction of this problem are exchanged within the Union of Laboratories and Specialists in Construction and Materials in Constructions.

\section{Conclusion}

This paper considers subjects dealing with the issues as the choice between the "long-term" and "rationalized| pavement construction and the problems in the assessment of layers of unbound granulated material or cement-connected stone material.

The concept of a long-lasting pavement construction, applied in highway construction during the eighties of the twentieth century, has been replaced by the strategy of low initial investment in the last cycle of highway construction. On sections where the pavement construction was designed according to the low initial cost strategy cracks due to material fatigue, accompanied by deformations appear as early as after the first period of exploitation. The bearing layers have low residual value so the damage and low level of comfort are related to low bearing capacity. The available design measures often include intrusion into the bearing layers or offer deep recycling toward the cold procedure as an alternative.

In our environment and at this moment no clear criteria have been established in the evaluation of the quality of the layer of non-connected granulated material in the highway pavement which is very often, and in conditions where "muniscule particles" are contaminated with plastic,clayish material makes it difficult to decide whether the optimal solution is to be sought in pavement reconstruction or not. This paper suggests the need to conduct studies including fundamental laboratory analyses.

In the previous cycle of road rehasbilitation and in the course of the current program, a reliable method of determination of the rigidity of the bearing layer of cement-stabilized granulated material. It can now be said that the reverse calculation of the module on the basis of measured deflections, by applying the linear elastic theory yield a clear picture about he rigidity and sopace homogeneity of the layer of cement-stabilized unbound granulated material. The designed rigidity of the layer of cement-stabilized bearing layer has a direct impact on the necessary thickness of the asphalt layers.

\section{References}

1. AASHTO Guide for the Design of Pavement Structures. Vol. 1-2, Amer. Assoc. Of State Highw. and Trans. Off. (1986)

2. AASHTO Guide for Design of Pavement Structures. Amer. Assoc. of State Highw. and Trans. Off. (1993)

3. C.R. Bennett, W.D.O. Paterson, A Guide to the Calibration and Adaptation, HDM - 4. Vol. 5, The Highw. Develop. and Manag. Ser., PIARC (2000)

4. E. Stannard, Getting Started with HDM-IV Version 2.0. The Highw. Develop. and Manag. Ser., University of Birmingham, UK (2005)

5. Z. Radojković, Pavement Management Systems. Belgrade (1990)

6. C.R. Bennett, W.D.O. Paterson, A Guide to the Calibration and Adaptation. HDM - 4. Vol. 5, The Highw. Develop. and Manag. Ser. (2000) 